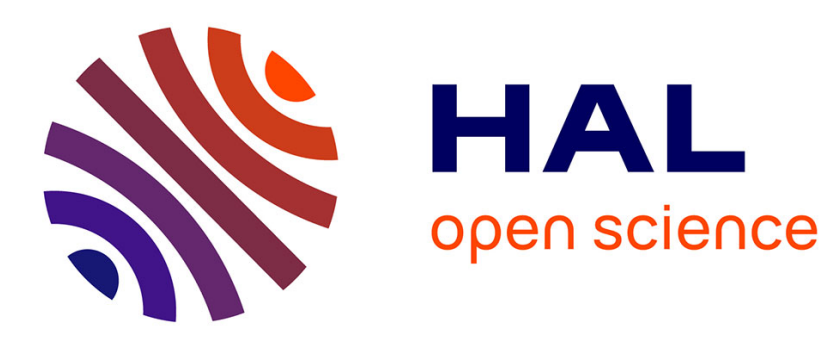

\title{
La polyarthrite rhumatoïde du sujet âgé
}

Adeline Ruyssen-Witrand

\section{To cite this version:}

Adeline Ruyssen-Witrand. La polyarthrite rhumatoïde du sujet âgé. Revue du Rhumatisme monographies, 2019, 86, pp.183 - 189. 10.1016/j.monrhu.2019.01.006 . hal-03484742

\section{HAL Id: hal-03484742 \\ https://hal.science/hal-03484742}

Submitted on 20 Dec 2021

HAL is a multi-disciplinary open access archive for the deposit and dissemination of scientific research documents, whether they are published or not. The documents may come from teaching and research institutions in France or abroad, or from public or private research centers.
L'archive ouverte pluridisciplinaire HAL, est destinée au dépôt et à la diffusion de documents scientifiques de niveau recherche, publiés ou non, émanant des établissements d'enseignement et de recherche français ou étrangers, des laboratoires publics ou privés.

\section{다)(1)}

Distributed under a Creative Commons Attribution - NonCommercial| 4.0 International 


\section{La polyarthrite rhumatoïde du sujet âgé}

\section{Rheumatoid arthritis in elderly}

Adeline Ruyssen-Witrand

Centre de rhumatologie, Hôpital Pierre Paul Riquet, CHU de Toulouse, 1 place du Dr Baylac, 31059 Toulouse cedex 09.

UMR 1027, Inserm, Université Paul Sabatier Toulouse III, Faculté de Médecine, 37 allées Jules Guesdes, 31000 Toulouse

Contact : ruyssen-witrand.a@chu-toulouse.fr; tel : 0561776976, télécopie : 0561777375 
Résumé

Du fait de l'augmentation de l'espérance de vie, la polyarthrite rhumatoïde (PR) du sujet fréquemment rencontrée en pratique courante avec une prévalence de l'ordre de $2 \%$. La présentation clinique initiale est souvent très aiguë avec des atteintes rhizoméliques et acroméliques associées et peut constituer un mode d'entrée dans la fragilité. Ces formes cliniques sont souvent de mauvais pronostics avec des altérations des capacités fonctionnelles et une progression structurale accélérée. Les stratégies de contrôle serré par objectif sont adaptées à la PR du sujet âgé et doivent permettre de limiter I'utilisation de glucocorticoïdes au long cours, principal facteur de complications cardiovasculaires et infectieuses, majorant le risque de fracture ostéoporotiques. La prescription de traitements de fond doit prendre en compte les interactions médicamenteuses éventuelles ainsi que des maladies associées. Les traitements de fond synthétiques ont un risque d'accumulation en cas d'insuffisance rénale et des adaptations de dose doivent être envisagées en cas d'altération de la fonction rénale. Les biomédicaments ont une efficacité comparable dans la PR à début tardif que la PR du sujet jeune et augmentent principalement le risque infectieux. Une évaluation multidisciplinaire gériatrique devrait être envisagée chez les patients à risque de fragilité.

Mots clés : Polyarthrite rhumatoïde, âge, début tardif

\section{Summary}

With the increased of life-expectancy, rheumatoid arthritis (RA) has become more frequent in routine practice with a prevalence of $2 \%$. Late-onset RA often begins with acute symptoms large and small joints and can lead to frailty. These clinical forms are often associated with poor functional and structural outcomes. The treat to target strategies remain valid and should aim to prevent from longterm glucocorticoid use, associated with cardiovascular events, infections and osteoporosis. Comorbidities and drug interactions should be considered when initiating a DMARD. A risk of accumulation because of kidney dysfunction can be observed with synthetic DMARDs and doses need to be adapted to renal function. bDMARDs have similar efficacy in late-onset RA compared to youngonset RA and increase infection risk. A geriatric multidisciplinary assessment should be considered in patients at risk of frailty.

Keywords : Rheumatoid arthritis, aged, late-onset, elderly 
La polyarthrite rhumatoïde (PR) est le rhumatisme inflammatoire chronique le plus fréquent chez l'adulte avec une prévalence autour de 0,5 à $1 \%$ en France. L'incidence de la PR augmente avec l'âge avec un pic d'incidence entre la $4^{\mathrm{e}}$ et la $6^{\mathrm{e}}$ décade [1]. Avec l'augmentation de l'espérance de vie dans les pays occidentaux, on note une augmentation du nombre de patients âgés atteints de PR avec une prévalence de l'ordre de $2 \%$ de la PR chez les sujets de plus de 60 ans [2].

L'Organisation Mondiale de la Santé (OMS) a proposé le seuil de 65 ans pour la définition du sujet âgé de seuil de 80 ans pour la définition de la personne très âgée. Ainsi les seuils de 60 et 65 ans sont très souvent utilisés dans la littérature pour identifier les populations âgées. Cependant, au niveau individuel, l'âge chronologique et l'âge fonctionnel ne coïncident pas avec un vieillissement différentiel des organes et des fonctions (Rapport mondial sur le vieillissement et santé http://apps.who.int/iris/bitstream/handle/10665/206556/9789240694842_fre.pdf?sequence=1). En effet, le vieillissement est synonyme de changements à la fois physiques, sociaux et psychologiques accompagnés d'un déclin fonctionnel progressif.

Il faut différentier ici la PR ayant débuté avant 60 ans chez un sujet vieillissant, de la PR à début tardif dont la présentation clinique, le pronostic et les diagnostics différentiels sont différents. Nous traiterons ici essentiellement de la PR à début tardif, décrit comme survenant après 60 ou 65 selon les études. Nous allons décrire les particularités cliniques des PR à début tardif, les précautions d'emploi lors de l'usage des traitements de la PR sur la population âgée, les points de surveillance des signes extra-articulaires et maladies associées sur cette population à risque et le risque de fragilité.

\section{Particularités cliniques de la PR du sujet âgé}

\subsection{Aspects cliniques de la $P R$ à début tardif}

L'incidence de la PR chez le sujet de plus de 60 ans a tendance à augmenter d'après les registres du début du XXlème siècle [3] et plus d'un tiers des PR surviendrait après l'âge de 60 ans [4]. Ces PR ont la particularité de toucher autant les petites que les grosses articulations et sont plus fréquemment associées au portage des allèles DRB1*13 et *14, tout comme le sont les patients atteints de pseudopolyarthrite rhizomélique (PPR) [5]. Ces PR touchent en effet souvent les ceintures scapulaires et pelviennes et peuvent être prises à tort pour des (PPR). Le sexe ratio est autour de 1,5 à 2 contre 4 à 4,5 pour les PR du sujet jeune. Les formes sont souvent très aigües accompagnées de signes généraux tels que la fièvre, l'amaigrissement, la fatigue, l'accélération de la vitesse de sédimentation. Elles sont moins souvent associées aux facteurs rhumatoïdes et ACPA [6]. On sépare classiquement les PR du 
sujet âgé en trois formes cliniques : la forme de type « polymyalgie rhumatoïde » qui représente $25 \%$ des cas et ressemble à une PPR, facteurs rhumatoïdes (FR) en général négatifs, souvent très aiguë initialement, avec une évolution bénigne sans érosions ; la forme œdémateuse, représentant $10 \%$ des cas, avec une évolution et progression proche du RS3PE (remitting seronegative symmetrical synovitis pitting oedema) ; la forme classique de PR érosive représente $70 \%$ des cas, à début typiquement brutal et associée à des signes généraux. Ces dernières formes ont un moins bon pronostic que chez les sujets jeunes avec un handicap fonctionnel plus important, une progression structurale plus rapide et des synovites actives en doppler plus nombreuses en échographie [7-9].

L'âge de début de la PR est un critère de mauvais pronostic fréquemment retrouvé dans la littérature. Les critères de rémission sont en effet moins souvent atteints dans la PR du sujet âgé, les scores HAQ (Health Assessment Questionnaire) sont en général plus élevés, surtout si le HAQ initial est élevé, la progression structurale est souvent plus importante $[7,9]$ et le risque de mortalité cardio-vasculaire est plus élevé dans cette population [10].

Il faut toutefois prendre en considération l'impact de l'âge sur les critères d'évaluation classique de la PR. En effet, la VS augmentant classiquement avec l'âge ce qui augmente naturellement la valeur de DAS28-VS pouvant expliquer des différences statistiquement significatives en termes de DAS28 ou de réponse EULAR dans les études et l'évaluation de l'activité de la PR doit préférentiellement être faite à l'aide du DAS28-CRP ou du SDAI[11]. D'autre part, le HAQ prenant en compte l'incapacité fonctionnelle des patients et l'appréciation globale de la maladie entrant dans le calcul du DAS28 peuvent être impactés par les conséquences directes du vieillissement sur la fonction, indépendamment de l'activité de la PR [11]. Enfin, il faudra tenir compte de la forte prévalence de l'arthrose digitale dans la population âgée pouvant fausser l'évaluation des scores structuraux classiques des études prenant en compte les pincements articulaires.

\subsection{Diagnostics différentiels}

Les principaux diagnostics différentiels de la PR à début tardif sont la PPR et le RS3PE. La présence d'auto-anticorps spécifiques et la recherche d'érosions radiologiques sont les éléments clés pour le diagnostic de PR. Toutefois, devant l'absence d'auto-anticorps ou d'érosions, d'autres examens complémentaires peuvent s'avérer utiles pour le diagnostic de PR. L'échographie peut en effet permettre de différentier une PR d'une PPR par la mise en évidence de synovites distales et symétriques avec signal doppler dans la PR, alors qu'on observe fréquemment des images de bursites des ceintures scapulaires et pelviennes dans la PPR [12]. Récemment, une étude a montré l'intérêt du 
PET-scanner pour distinguer une PR d'une PPR avec une sensibilité et une spécificité de l'ordre de $86 \%$ [13].

Le RS3PE est un rhumatisme inflammatoire caractéristique de la personne âgée qui se manifeste par l'apparition brutale d'arthrites périphériques symétriques associées à des œdèmes touchant les mains et poignets et/ou les chevilles et les pieds. Ces œdèmes sont en rapport avec des teno-synovites des tendons extenseurs des mains et des pieds. Les RS3PE ne développent pas d'érosions et n'ont pas de facteurs rhumatoïdes. Le pronostic est excellent avec une très grand corticosensibilité [14].

Les autres diagnostics différentiels à envisager classiquement chez le sujet âgé sont les arthropathies microcristallines, les spondyloarthrites, la polyarthrose, les connectivites et vascularites, les syndromes paranéoplasiques, souvent satellites d'hémopathies, l'ostéoarthropathie hypertrophique pneumique ou syndrome de Pierre-Marie et Foix, la sarcoïdose et les arthrites infectieuses (VHB, VHC, VIH, Parvovirus B19 et autres) et l'endocardite [14].

\section{Traitements de la PR du sujet âgé}

Bien qu'ayant une présentation clinique inaugurale souvent plus aiguë et un plus mauvais pronostic fonctionnel et structural, la PR à début tardif reçoit souvent des traitements moins " agressifs » en comparaison à la PR du sujet jeune [15], l'âge supérieur à 75 ans étant un facteur de non prescription de traitement de fond reconnu [16]. Ceci est souvent justifié par les insuffisances rénales ou hépatiques plus fréquentes, la présence de polymédication augmentant le risque d'interactions médicamenteuses, des comorbidités et du risque infectieux augmenté. Toutefois, cette population reste plus souvent exposée aux glucocorticoïdes[15, 17].

Les AINS doivent être utilisés de manière prudente en tenant compte de la fonction rénale, du risque d'interaction médicamenteuse, du risque augmenté d'effets indésirables cardio-vasculaires, rénaux et gastro-intestinaux. Compte tenu du risque cardio-vasculaire augmenté dans cette population, les coxibs et le diclofenac ne devraient pas être utilisés en première intention (règle de bon usage des AINS,

ANSM

2013 https://ansm.sante.fr/var/ansm_site/storage/original/application/53960970b52f1b0c30da77518e8c 86d7.pdf) [18]. II faut noter que deux études ont récemment montré que le risque cardiovasculaire 
sous coxib était comparable à celui des patients sous naproxène, raison pour laquelle tous les AINS devraient être évités en cas de facteurs de risque cardiovasculaire dans cette population à risque [19, 20]. Un contrôle de du débit de filtration glomérulaire (CKD-EPI) et de la kaliémie peut être souhaitable après l'initiation d'un AINS chez les patients ayant une altération de la fonction rénale ou recevant des traitements ayant un impact sur la fonction rénale (inhibiteurs de l'enzyme de conversion, antagonistes de l'angiotensine, diurétiques). Enfin, il est de bon usage de ne prescrire les AINS que sur une période courte, à dose minimale efficace, en privilégiant les formes à demi-vie courte

Les glucocorticoïdes prescrits à faible dose (7,5mg à $10 \mathrm{mg} / \mathrm{j}$ d'équivalent prednisone) augmentent les taux de rémission et diminuent à moyen terme le risque d'évolution structurale dans la PR débutante [21-23]. Ils exposent toutefois les patients au risque de complications multiples telles que le diabète, I'HTA, l'ostéoporose, la cataracte, le glaucome, la fragilité cutanée, le risque infectieux...etc. Le risque infectieux notamment est augmenté même avec les faibles doses et persiste plusieurs mois après I'arrêt de glucocorticoïdes[17]. Ils sont privilégiés aux AINS en traitement symptomatique des poussées chez les patients âgés mais la prescription doit être si possible limitée dans le temps. La prednisone est privilégiée à la prednisolone car a une variabilité interindividuelle moindre de sa pharmacocinétique II convient d'éviter l'interchangeabilité entre le Cortancyl ${ }^{\circledR}$ princeps et les génériques du fait de variabilité de biodisponibilité entre les présentations [22].

\subsection{Traitements de fond conventionnels}

Le méthotrexate reste le traitement de première intention, même après 60 ans, et son efficacité sur cette population a déjà été largement rapportée. Cependant, il est souvent mal toléré à cet âge avec des effets indésirables rapportés par 30 à $50 \%$ des patients [24]. Le risque principal sur cette population et celui de l'augmentation de la toxicité hépatique, hématologique ou pulmonaire du fait d'une accumulation des métabolites du méthotrexate, liée à l'altération de la filtration glomérulaire fréquente à cet âge, la dénutrition et l'hypoalbuminémie en rapport avec le syndrome inflammatoire. Le traitement par méthotrexate n'est pas recommandé lorsque la clairance de la créatinine est inférieure à $15 \mathrm{ml} / \mathrm{min}$ et une adaptation posologique est recommandée pour ceux dont la clairance est comprise entre 15 et $60 \mathrm{ml} / \mathrm{min}$. Certains recommandent d'initier le méthotrexate à une posologie comprise entre 2,5 et $7,5 \mathrm{mg} / \mathrm{sem}$ et d'augmenter prudemment jusqu'à $12,5 \mathrm{mg} / \mathrm{sem}$ maximum lorsque la clairance est comprise entre 25 et $60 \mathrm{ml} / \mathrm{min}$ [25]. Les patients âgés étant souvent carencés, une supplémentation en acide folique doit être systématiquement proposée pour diminuer le risque 
de toxicité médullaire et une recharge en folates avant l'initiation du méthotrexate doit être prescrite en cas de carence.

La tolérance de la sulfsalazine et du leflunomide semble comparable chez les personnes âgées en comparaison aux sujets sains. L'utilisation de l'hydroxychloroquine doit être prudente chez les patients âgés avec altération du débit de filtration glomérulaire car celui-ci est un facteur de toxicité rétinienne [26] . La combinaison méthotrexate-hydroxychloroquine peut être particulièrement pertinente car I'hydroxychloroquine augmente la biodisponibilité du méthotrexate, limite les effets secondaires notamment hépatiques et améliore les syndromes métaboliques et le risque cardiovasculaire [27, 28].

\subsection{Traitements de fond biologiques et thérapies ciblées}

\subsubsection{Efficacité des agents biologiques chez le sujet âgé}

Les données d'efficacité et de tolérance sont rarement disponibles dans les essais thérapeutiques chez les sujets de plus de 75 ans. Plusieurs registres européens ont évalué l'impact de l'âge sur la réponse thérapeutique aux agents biologiques (bDMARDs pour biologic Disease Modifying Anti-Rheumatic Drugs).

Les anti-TNF ont une efficacité sensiblement comparable lorsqu'on compare les sujets jeunes aux sujets de plus de 60 ans en prenant les critères de réponse classique (ACR20, ACR50) [29, 30]. Les scores HAQ sont en général moins améliorés chez les personnes de plus de 75 ans, ce qui est expliqué par l'impact des conséquences du vieillissement lui-même sur ce score fonctionnel. Les analyses posthoc des essais randomisés portant sur les anti-TNF (etanercept, infliximab, adalimumab) ont rapporté une efficacité de ces molécules pour améliorer les signes et symptômes des patients, la fonction et ont démontré leur capacité à réduire la progression structurale, surtout lorsqu'ils sont prescrits en association avec le méthotrexate chez les sujets âgés, avec des taux de réponses comparables aux patients plus jeunes [31].

Les données d'efficacité des autres agents biologiques sur cette population âgée sont plus rares. Dans le registre ORA, 103 patients de plus de 75 ans et 215 patients de 65 à 74 ans ont été comparés aux patients plus jeunes. Ces patients avaient lors de l'inclusion dans le registre une durée d'évolution plus longue et une maladie plus active avec des DAS28 et des CRP plus élevés. Globalement, les taux de réponse EULAR étaient comparables selon les groupes et une variation du DAS28 à 24 mois comparable [32]. L'efficacité de l'abatacept a également été évaluée dans un registre japonais de 500 PR et n'a pas 
démontré de différence significative en termes de réponse EULAR ou de maintien thérapeutique à 3 ans entre les patients âgés de plus de 72 ans, ceux âgés de 62 à 71 ans et les plus jeunes [33].

Dans le registre AIR, seuls $7 \%$ des patients de plus de 75 ans avaient une bonne réponse EULAR au rituximab (comparé à 22 à $26 \%$ de bonne réponse chez les patients plus jeunes) à un an. II faut noter que dans les items du DAS28, il n'était pas observé de différence significative en termes d'amélioration du nombre d'articulations gonflées, douloureuses et appréciation globale de l'activité de la maladie entre les patients de plus de 75 ans et ceux plus jeunes, mais une moins grande décroissance de la VS chez les plus de 75 ans [34].

L'efficacité du tocilizumab a été évaluée dans une étude française rétrospective de 222 PR dont 61 de plus de 65 ans. La bonne réponse EULAR était moins souvent obtenue chez les patients de plus de 65 ans (41\% versus $61 \%, p<0,01$ ), les taux de maintien thérapeutique étaient en revanche similaires [35]. Bien qu'ayant une efficacité sensiblement comparable chez les personnes âgées en comparaison aux sujets plus jeunes, l'utilisation des bDMARDs est moins fréquente chez les patients ayant une PR à début tardif alors que l'utilisation de glucocorticoïdes est plus répandue chez les sujets dont la PR a un début tardif $[4,15]$.

\subsubsection{Tolérance des agents biologiques chez les sujets âgés}

Le vieillissement est souvent accompagné du développement de comorbidités notamment cardiovasculaires, infectieuses et néoplasiques. Ainsi, les effets indésirables graves rapportés dans les essais thérapeutiques et les registres surviennent plus fréquemment chez les sujets âgés, plus exposés naturellement à ces risques de complication. Ceci est particulièrement vrai dans la PR, qui augmente elle-même le risque infectieux, avec surtout des infections respiratoires, des zonas et des infections des parties molles fréquemment observées dans les populations PR de plus de 66 ans.

Les facteurs associés au risque infectieux sont la présence de comorbidités associées, notamment le diabète, les antécédents d'infection sévère, l'utilisation d'agents biologiques et surtout de glucocorticoïdes, risque restant élevé, même si le sevrage remonte à plus de 2 ans [17].

Les registres montrent une augmentation du risque infectieux plus importante chez les sujets âgés sous bDMARDs comparés aux plus jeunes. La prise d'anti-TNF augmente ainsi de $20 \%$ le risque d'infection chez les sujets âgés. Bien que plusieurs études sur registre aient suggéré que la prise du récepteur soluble du TNF était associée à un moindre risque infectieux en comparaison à certains anticorps monoclonaux ciblant le TNF [36, 37], ceci n'a pas été démontré dans une étude portant sur 
population de plus de 65 ans [38]. Une analyse sur les données de Medicare aux Etats Unis, des patients de plus de 65 ans a comparé le risque de réhospitalisation pour infection après une première infection hospitalisée en fonction de la prise de divers bDMARDs. L'incidence des réhospitalisation dans cette population à risque était de l'ordre de 15,3/100 patients-années. Après ajustement sur un score de risque infectieux et autres facteurs confondants, I'infliximab (HR [IC95\%] =1,39 [1,21-1,53]), le rituximab (HR [IC95\%] = 1.36 [1.21-1.53]) et l'etanercept (HR [IC95\%] = 1.24[1.07-1.45]) étaient associés à un plus grand risque de réhospitalisation en comparaison avec l'abatacept [39].

Les anti-TNF ont été décrits, pour certains comme à risque de décompensation d'insuffisance cardiaque, voire pourraient induire une insuffisance cardiaque de novo. Toutefois, les données de la littérature restent contradictoires suggérant parfois une diminution du risque sous anti-TNF en comparaison aux traitements de fond conventionnels. Cependant, beaucoup d'études ne mentionnent pas la co-prescription de glucocorticoïdes, ce qui rend difficile l'interprétation des associations entre la prise d'anti-TNF et d'insuffisance cardiaque rapportées [40]. En cas de signes cliniques faisant suspecter une insuffisance cardiaque, il convient d'évaluer la fonction ventriculaire par la mesure de la fraction d'éjection. II n'est pas recommandé d'initier un anti-TNF en cas d'insuffisance cardiaque modérée à sévère (grade III et IV). Une étude récente semble suggérer que le risque cardiovasculaire est plus faible chez les patients sous abatacept comparés aux patients recevant d'autres agents biologiques chez les patients de Medicare[41].

\subsection{Inhibiteurs de JAK et personne âgée}

Deux études post-hoc d'essais industriels ont évalué l'efficacité du baricitinib [42] et du tofacitinib [43] chez les patients de plus de 65 ans en comparaison aux sujets plus jeunes. Les taux d'efficacité des inhibteurs de JAK étaient comparables à ceux des patients de moins de 65 ans. Les événements indésirables étaient, comme attendu, plus nombreux chez les patients de plus de 65 ans, en particulier les infections zostériennes [42].

Les inhibiteurs de JAK sont principalement éliminés par filtration glomérulaire. Les études de pharmacocinétiques n'ont pas montré d'effet sur la pharmacocinétique du baricitinib chez les sujets âgés de 65 à 75 ans. L'expérience clinique étant limitée chez les plus de 75 ans, il est recommandé d'initier le baricitinib à la posologie de $2 \mathrm{mg} / \mathrm{j}$ chez les 75 ans et plus et chez les patients ayant une clairance de la créatinine comprise entre 30 et $60 \mathrm{ml} / \mathrm{min}$. Le baricitinib n'est pas recommandé lorsque la clairance de la créatinine est inférieure à $30 \mathrm{ml} / \mathrm{min}$ (Résumé des caractéristiques du produit). 
Les données cliniques sont également limitées pour l'utilisation du tofacitinib chez les patients de plus de 75ans. Cependant, aucun ajustement de dose n'est préconisé chez les sujets âgés, ni en cas d'insuffisance rénale légère à modérée (clairance au-dessus de $30 \mathrm{ml} / \mathrm{min}$ ). Les patients ayant une insuffisance rénale sévère (clairance $<30 \mathrm{ml} / \mathrm{min}$ ) doivent prendre une dose réduite à $5 \mathrm{mg} / \mathrm{j}$, y compris après dialyse (Résumé des caractéristiques du produit).

Les données d'extensions des essais cliniques du tofacitinib ont identifié un taux d'infections sévères de 2,7/100 PA, et un taux de zona autour de 3,9/100PA [44]. L'âge et l'utilisation des glucocorticoïdes sont les facteurs classiques de survenue de zona.

\subsection{Stratégie par objectif dans la $P R$ à début tardif}

Il est recommandé d'adapter les traitements de fond en se fixant comme objectif la rémission, ou à défaut le bas niveau d'activité [1]. La recherche de rémission en utilisant les critères stringents proposés par l'EULAR peut parfois conduire à des escalades thérapeutiques tout en exposant les patients à des risques d'effets indésirables. Un bas niveau d'activité DAS28 est un objectif alternatif qui pourrait être appliqué à la personne âgée. Dans une cohorte prospective de 151 PR à début tardif [45], récentes, dont l'âge moyen était de 75ans, l'adhérence à une stratégie de type traitement par objectif était estimée à $84 \%$ à 6 mois et $76 \%$ à 1 an. Parmi ces patients, $2 / 3$ recevaient du méthotrexate en monothérapie et $1 / 3$ un biomédicament (majoritairement un anti-TNF). Le bas niveau d'activité selon le DAS28 était obtenu pour la moitié des patients, $50 \%$ des patients n'avaient pas de progression structurale significative et $63 \%$ avaient un score $H A Q \leq 5$ (rémission fonctionnelle). Ainsi les recommandations actuelles semblent aussi adaptées sur cette population et le critère bas niveau d'activité un objectif réaliste.

\section{Prise en charge des atteintes extra-articulaires et maladies}

\section{associées}

Les patients âgés ont fréquemment des comorbidités qui conditionnent fortement le choix des traitements. 


\subsection{Maladies cardiovasculaires}

L'athérosclérose est une conséquence fréquente du vieillissement et est augmentée dans la PR, constituant ainsi la principale cause de décès. Un registre a montré que le risque de maladie coronarienne, d'infarctus du myocarde, d'accidents vasculaires cérébraux et d'hypertension était plus élevé dans la PR à début tardif en comparaison aux PR ayant débuté plus précocement, après ajustement sur l'âge [15]. Dans un registre suédois, le risque d'évènements cardiovasculaires et la mortalité étaient augmentés chez les sujets ayant une PR à début tardif, ce risque était diminué en cas de bon contrôle de l'activité de la maladie et de la fonction, alors que la prise de glucocorticoïdes augmentait ce risque [46]. D'après les recommandations EULAR, les PR avec facteurs de risque cardiovasculaires devraient bénéficier de dépistage précoce et de traitements plus intensifs afin de diminuer le risque de mortalité d'origine cardiovasculaire [47] .

\subsection{Risque infectieux}

Le risque infectieux est plus élevé après 65 ans et augmente avec l'âge. La PR est caractérisée par une accélération des signes d'immunosénescence, indépendamment de la durée de la maladie ou de l'âge de début, affectant à la fois l'immunité innée et adaptative et diminuant ainsi les capacités à résoudre les infections [14]. Le risque infectieux est également lié à la iatrogénie. Le traitement exposant le plus fréquemment au risque infectieux est la prise de glucocorticoïdes au long cours, risque qui reste élevé même en cas de sevrage datant de plus de 2 ans [17]. II faut noter que ces patients sont particulièrement exposés au risque de zona, risque favorisé également par la prise de glucocorticoïdes et des différents traitements de fond de la PR [48].

La vaccination anti-grippale devrait être systématiquement réalisée après 65 ans et a démontré son efficacité en réduisant la mortalité d'environ 40 \%[49]. Il est conseillé d'interrompre le méthotrexate pendant 2 semaines après la vaccination, pour favoriser l'immunisation [50]. Bien que la réponse vaccinale après vaccination anti-pneumococcique soit altérée par l'utilisation du méthotrexate en combinaison par anti-TNF ou tocilizumab et par le tofacitinib, cette vaccination est reste recommandée en pratique courante [47].

Le vaccin contre le zona est indiqué chez les patients de plus de 65 ans. Cependant, il s'agit d'un vaccin vivant qui reste contre-indiqué en cas de prise de méthotrexate, de biomédicament ou thérapies ciblées ou de glucocorticoïdes au long cours à plus de $10 \mathrm{mg} / \mathrm{j}$. Il est probable que le prochain vaccin anti-zona inactivé sera recommandé dans cette population à risque. 


\subsection{Pneumopathies interstitielles diffuses (PID)}

Les PID, manifestation extra-articulaire des PR, sont associées à une augmentation du risque de mortalité. Ce risque augmente avec l'âge et la médiane de survie après diagnostic de PID est de 2,6 ans. La PID augmente le risque d'infection pulmonaire, ce d'autant que l'utilisation de glucocorticoïdes est fréquente chez ces patients. Compte tenu de la prévalence augmentée de PID chez les sujets âgés, la prescription de scanner pulmonaire devrait être envisagée en cas de signes fonctionnels pulmonaires ou d'anomalies à l'auscultation pulmonaire [47].

\subsection{Risque de cancer}

Les sujets âgés atteints de PR sont particulièrement à risque de développer un cancer solide ou une hémopathie. L'immunosénescence accélérée en cas dans la PR favorise l'émergence de néoplasies profondes et d'hémopathies. Les méta-analyses et les données de registres n'ont pas montré de surrisque de maladie néoplasique en cas d'utilisation de biomédicaments, en dehors des tumeurs cutanées. Les dépistages systématiques des cancers recommandés par la HAS et le dépistage systématique des tumeurs cutanées par le dermatologue doivent être encouragés chez ces patients à risque [47].

\subsection{Risque d'ostéoporose}

La PR est un facteur de risque indépendant de sur venue de fractures ostéoporotiques, risque majoré avec l'âge, I'utilisation de glucocorticoïdes, la diminution des activités physiques, l'augmentation du risque de chutes. Un dépistage systématique de l'ostéoporose est recommandé chez les patients atteints de PR [47]. Le risque de fracture peut être diminué par un dépistage précoce de l'ostéoporose et traitement le cas échéant, en évitant l'utilisation de glucocorticoïdes au long cours, en favorisant I'activité physique chez les personnes âgées atteintes de PR.

\subsection{Prise en charge de la fragilité}


La fragilité est un concept gériatrique et est définie par des modifications dans 5 grands domaines comprenant la perte de poids (ou sarcopénie), ralentissement de la marche, asthénie, faiblesse musculaire et bas niveau d'activité physique. Un patient est considéré comme fragile en cas d'altération d'au moins 3 de ces 5 domaines. Un patient ayant une altération de 1 ou 2 de ces domaines est considéré comme pré-fragile et un patient n'ayant aucune altération dans ces domaines est classé en « robuste » ou « non-fragile ». Il existe de façon associée des altérations dans les activités sociales, des modifications psychologiques et de la fonction physique qui varient en fonction du degré de fragilité. Les comorbidités telles que l'arthrose, les maladies pulmonaires, les maladies cardiovasculaires, les accidents vasculaires cérébraux, l'insuffisance rénale, la sarcopénie, la dépression et les troubles cognitifs sont les facteurs de risque majeurs de sarcopénie. Les personnes en situation de fragilité sont particulièrement exposées au risque de déclin de la fonction, de chutes, d'institutionnalisation, d'escarres, de troubles psychiatriques, d'incontinence, de malnutrition et de mortalité [45].

Les patients âgés atteints de PR sont particulièrement à risque de fragilité du fait des altérations fonctionnelles liées à la maladie, de la dépression, des troubles cognitifs, des chutes, de la malnutrition et de la polymédication. Afin de prévenir la survenue de "syndrome gériatrique " irréversible (comprenant notamment une incapacité à réaliser ses activités quotidiennes, des troubles de la vue, des troubles cognitifs, des troubles de l'audition, l'incontinence urinaire, la diminution des activités sociales), les patients débutant tardivement une PR devraient bénéficier de thérapeutiques intensives afin de les ramener dans la pré-fragilité ou la non-fragilité (figure 1). L'activité physique a démontré son efficacité en termes de réduction du risque cardio-vasculaire et d'ostéoporose devrait être promue chez ces patients à risque. Enfin, compte tenu de la complexité de la prise en charge de la PR sur cette population du fait des comorbidités associées et de la polymédication, des évaluations multidisciplinaires gériatriques devraient être organisées, afin de prévenir le risque iatrogénique et les conséquences fonctionnelles de la PR chez le sujet âgé [45].

\section{Conclusion}

La PR du sujet âgé est une situation fréquemment rencontrée en pratique courante. L'évaluation des comorbidités et du risque de fragilité doit être soigneuse et prise en compte lors de choix des traitements. L'utilisation des AINS doit être évitée en privilégiant un traitement symptomatique par glucocorticoïdes dont la prescription doit être limitée dans le temps. Les recommandations générales de prise en charge de la PR restent adaptées dans cette population de plus de 65 ans en tenant compte 
de la fragilité de ces patients. Compte tenu du peu de données dans la littérature, des études de stratégies sont encore nécessaires pour guider le clinicien dans ses choix thérapeutiques chez les sujets de plus de 75 ans.

Conflits d'intérêts: I'auteur ne déclare aucun conflit d'intérêt pour cet article. 
[1] Daien C, Hua C, Gaujoux-Viala C, Cantagrel A, Dubremetz M, Dougados M, et al. Update of French Society for Rheumatology Recommendations for Managing Rheumatoid Arthritis. Joint Bone Spine 2018.

[2] Rasch EK, Hirsch R, Paulose-Ram R, Hochberg MC. Prevalence of rheumatoid arthritis in persons 60 years of age and older in the United States: effect of different methods of case classification. Arthritis Rheum 2003;48(4):917-26.

[3] Nikiphorou E, Norton S, Carpenter L, Dixey J, Andrew Walsh D, Kiely P, et al. Secular Changes in Clinical Features at Presentation of Rheumatoid Arthritis: Increase in Comorbidity But Improved Inflammatory States. Arthritis Care Res (Hoboken) 2017;69(1):21-7.

[4] Mueller RB, Kaegi T, Finckh A, Haile SR, Schulze-Koops H, von Kempis J, et al. Is radiographic progression of late-onset rheumatoid arthritis different from young-onset rheumatoid arthritis? Results from the Swiss prospective observational cohort. Rheumatology (Oxford) 2014;53(4):671-7.

[5] Wu H, Khanna D, Park G, Gersuk V, Nepom GT, Wong WK, et al. Interaction between RANKL and HLA-DRB1 genotypes may contribute to younger age at onset of seropositive rheumatoid arthritis in an inception cohort. Arthritis Rheum 2004;50(10):3093-103.

[6] Spinel-Bejarano N, Quintana G, Heredia R, Yunis JJ, Caminos JE, Garces MF, et al. Comparative study of elderly-onset rheumatoid arthritis and young-onset rheumatoid arthritis in a Colombian population: clinical, laboratory and HLA-DRB1 findings. Clin Exp Rheumatol 2013;31(1):40-6.

[7] Arnold MB, Bykerk VP, Boire G, Haraoui BP, Hitchon C, Thorne C, et al. Are there differences between young-and older-onset early inflammatory arthritis and do these impact outcomes? An analysis from the CATCH cohort. Rheumatology (Oxford) 2014;53(6):1075-86.

[8] Dejaco C, Duftner C, Wipfler-Freissmuth E, Weiss H, Schneider T, Schirmer M. Elderly- versus younger-onset rheumatoid arthritis: higher levels of ultrasound-detected inflammation despite comparable clinical disease activity. Arthritis Care Res (Hoboken) 2013;65(2):304-8.

[9] Krams T, Ruyssen-Witrand A, Nigon D, Degboe Y, Tobon G, Fautrel B, et al. Effect of age at rheumatoid arthritis onset on clinical, radiographic, and functional outcomes: The ESPOIR cohort. Joint Bone Spine 2016;83(5):511-5.

[10] Naz SM, Farragher TM, Bunn DK, Symmons DP, Bruce IN. The influence of age at symptom onset and length of followup on mortality in patients with recent-onset inflammatory polyarthritis. Arthritis Rheum 2008;58(4):985-9.

[11] Ito H, Ogura T, Hirata A, Takenaka S, Mizushina K, Fujisawa Y, et al. Global assessments of disease activity are age-dependent determinant factors of clinical remission in rheumatoid arthritis. Semin Arthritis Rheum 2017;47(3):310-4.

[12] Weigand S, Ehrenstein B, Fleck M, Hartung W. Joint involvement in patients with early polymyalgia rheumatica using high-resolution ultrasound and its contribution to the EULAR/ACR 2012 classification criteria for polymyalgia rheumatica. J Rheumatol 2014;41(4):730-4.

[13] Wakura D, Kotani T, Takeuchi T, Komori T, Yoshida S, Makino S, et al. Differentiation between Polymyalgia Rheumatica (PMR) and Elderly-Onset Rheumatoid Arthritis Using 18FFluorodeoxyglucose Positron Emission Tomography/Computed Tomography: Is Enthesitis a New Pathological Lesion in PMR? PLoS One 2016;11(7):e0158509.

[14] Villa-Blanco JI, Calvo-Alen J. Elderly onset rheumatoid arthritis: differential diagnosis and choice of first-line and subsequent therapy. Drugs Aging 2009;26(9):739-50.

[15] Tutuncu Z, Reed G, Kremer J, Kavanaugh A. Do patients with older-onset rheumatoid arthritis receive less aggressive treatment? Ann Rheum Dis 2006;65(9):1226-9.

[16] Solomon DH, Yelin E, Katz JN, Lu B, Shaykevich T, Ayanian JZ. Treatment of rheumatoid arthritis in the Medicare Current Beneficiary Survey. Arthritis Res Ther 2013;15(2):R43. 
[17] Dixon WG, Abrahamowicz M, Beauchamp ME, Ray DW, Bernatsky S, Suissa S, et al. Immediate and delayed impact of oral glucocorticoid therapy on risk of serious infection in older patients with rheumatoid arthritis: a nested case-control analysis. Ann Rheum Dis 2012;71(7):1128-33.

[18] Schmidt M, Sorensen HT, Pedersen L. Diclofenac use and cardiovascular risks: series of nationwide cohort studies. BMJ 2018;362:k3426.

[19] Bally M, Dendukuri N, Rich B, Nadeau L, Helin-Salmivaara A, Garbe E, et al. Risk of acute myocardial infarction with NSAIDs in real world use: bayesian meta-analysis of individual patient data. BMJ 2017;357:j1909.

[20] Nissen SE, Yeomans ND, Solomon DH, Luscher TF, Libby P, Husni ME, et al. Cardiovascular Safety of Celecoxib, Naproxen, or Ibuprofen for Arthritis. N Engl J Med 2016;375(26):251929.

[21] Kirwan JR, Bijlsma JW, Boers M, Shea BJ. Effects of glucocorticoids on radiological progression in rheumatoid arthritis. Cochrane Database Syst Rev 2007(1):CD006356.

[22] Lega J, Durupt S, Reynaud Q, Vital-Durand D, Durieu S. Controverse dans la corticothérapie des vascularites systémiques. Revue du rhumatisme Monographies 2017;84(3):262-9.

[23] Svensson B, Ahlmen M, Forslind K. Treatment of early RA in clinical practice: a comparative study of two different DMARD/corticosteroid options. Clin Exp Rheumatol 2003;21(3):32732.

[24] Jin XM, Lee J, Choi NK, Seong JM, Shin JY, Kim YJ, et al. Utilization patterns of diseasemodifying antirheumatic drugs in elderly rheumatoid arthritis patients. J Korean Med Sci 2014;29(2):210-6.

[25] Karie S, Gandjbakhch F, Janus N, Launay-Vacher V, Rozenberg S, Mai Ba CU, et al. Kidney disease in RA patients: prevalence and implication on RA-related drugs management: the MATRIX study. Rheumatology (Oxford) 2008;47(3):350-4.

[26] Kim JW, Kim YY, Lee H, Park SH, Kim SK, Choe JY. Risk of Retinal Toxicity in Longterm Users of Hydroxychloroquine. J Rheumatol 2017;44(11):1674-9.

[27] Carmichael SJ, Beal J, Day RO, Tett SE. Combination therapy with methotrexate and hydroxychloroquine for rheumatoid arthritis increases exposure to methotrexate. J Rheumatol 2002;29(10):2077-83.

[28] Rempenault C, Combe B, Barnetche T, Gaujoux-Viala C, Lukas C, Morel J, et al. Metabolic and cardiovascular benefits of hydroxychloroquine in patients with rheumatoid arthritis: a systematic review and meta-analysis. Ann Rheum Dis 2018;77(1):98-103.

[29] Hetland ML, Christensen IJ, Tarp U, Dreyer L, Hansen A, Hansen IT, et al. Direct comparison of treatment responses, remission rates, and drug adherence in patients with rheumatoid arthritis treated with adalimumab, etanercept, or infliximab: results from eight years of surveillance of clinical practice in the nationwide Danish DANBIO registry. Arthritis Rheum 2010;62(1):22-32.

[30] Radovits BJ, Fransen J, van Riel PL, Laan RF. Influence of age and gender on the 28-joint Disease Activity Score (DAS28) in rheumatoid arthritis. Ann Rheum Dis 2008;67(8):1127-31.

[31] Bathon JM, Fleischmann RM, Van der Heijde D, Tesser JR, Peloso PM, Chon Y, et al. Safety and efficacy of etanercept treatment in elderly subjects with rheumatoid arthritis. J Rheumatol 2006;33(2):234-43.

[32] Lahaye C, Soubrier M, Mulliez A, Bardin T, Cantagrel A, Combe B, et al. Effectiveness and safety of abatacept in elderly patients with rheumatoid arthritis enrolled in the French Society of Rheumatology's ORA registry. Rheumatology (Oxford) 2016;55(5):874-82.

[33] Takahashi N, Kojima T, Kida D, Kaneko A, Hirano Y, Fujibayashi T, et al. Clinical effectiveness and long-term retention of abatacept in elderly rheumatoid arthritis patients: Results from a multicenter registry system. Mod Rheumatol 2018:1-23.

[34] Payet S, Soubrier M, Perrodeau E, Bardin T, Cantagrel A, Combe B, et al. Efficacy and safety of rituximab in elderly patients with rheumatoid arthritis enrolled in a French Society of Rheumatology registry. Arthritis Care Res (Hoboken) 2014;66(9):1289-95. 
[35] Pers YM, Schaub R, Constant E, Lambert J, Godfrin-Valnet M, Fortunet C, et al. Efficacy and safety of tocilizumab in elderly patients with rheumatoid arthritis. Joint Bone Spine 2015;82(1):25-30.

[36] Curtis JR, Yang S, Patkar NM, Chen L, Singh JA, Cannon GW, et al. Risk of hospitalized bacterial infections associated with biologic treatment among US veterans with rheumatoid arthritis. Arthritis Care Res (Hoboken) 2014;66(7):990-7.

[37] van Dartel SA, Fransen J, Kievit W, Flendrie M, den Broeder AA, Visser H, et al. Difference in the risk of serious infections in patients with rheumatoid arthritis treated with adalimumab, infliximab and etanercept: results from the Dutch Rheumatoid Arthritis Monitoring (DREAM) registry. Ann Rheum Dis 2013;72(6):895-900.

[38] Galloway JB, Hyrich KL, Mercer LK, Dixon WG, Fu B, Ustianowski AP, et al. Anti-TNF therapy is associated with an increased risk of serious infections in patients with rheumatoid arthritis especially in the first 6 months of treatment: updated results from the British Society for Rheumatology Biologics Register with special emphasis on risks in the elderly. Rheumatology (Oxford) 2011;50(1):124-31.

[39] Yun H, Xie F, Delzell E, Chen L, Levitan EB, Lewis JD, et al. Risk of hospitalised infection in rheumatoid arthritis patients receiving biologics following a previous infection while on treatment with anti-TNF therapy. Ann Rheum Dis 2015;74(6):1065-71.

[40] Tocci G, Goletti D, Marino V, Matucci A, Milano GM, Cantini F, et al. Cardiovascular outcomes and tumour necrosis factor antagonists in chronic inflammatory rheumatic disease: a focus on rheumatoid arthritis. Expert Opin Drug Saf 2016;15(sup1):55-61.

[41] Zhang J, Xie F, Yun H, Chen L, Muntner P, Levitan EB, et al. Comparative effects of biologics on cardiovascular risk among older patients with rheumatoid arthritis. Ann Rheum Dis 2016;75(10):1813-8.

[42] Fleischmann R, Alam J, Arora V, Bradley J, Schlichting DE, Muram D, et al. Safety and efficacy of baricitinib in elderly patients with rheumatoid arthritis. RMD Open 2017;3(2):e000546.

[43] Curtis JR, Schulze-Koops H, Takiya L, Mebus CA, Terry KK, Biswas P, et al. Efficacy and safety of tofacitinib in older and younger patients with rheumatoid arthritis. Clin Exp Rheumatol 2017;35(3):390-400.

[44] Cohen SB, Tanaka Y, Mariette X, Curtis JR, Lee EB, Nash P, et al. Long-term safety of tofacitinib for the treatment of rheumatoid arthritis up to 8.5 years: integrated analysis of data from the global clinical trials. Ann Rheum Dis 2017;76(7):1253-62.

[45] Sugihara T, Harigai M. Targeting Low Disease Activity in Elderly-Onset Rheumatoid Arthritis: Current and Future Roles of Biological Disease-Modifying Antirheumatic Drugs. Drugs Aging 2016;33(2):97-107.

[46] Ajeganova S, Andersson ML, Frostegard J, Hafstrom I. Disease factors in early rheumatoid arthritis are associated with differential risks for cardiovascular events and mortality depending on age at onset: a 10-year observational cohort study. J Rheumatol 2013;40(12):1958-66.

[47] Baillet A, Gossec L, Carmona L, Wit M, van Eijk-Hustings $Y$, Bertheussen $H$, et al. Points to consider for reporting, screening for and preventing selected comorbidities in chronic inflammatory rheumatic diseases in daily practice: a EULAR initiative. Ann Rheum Dis 2016;75(6):965-73.

[48] Pappas DA, Hooper MM, Kremer JM, Reed G, Shan Y, Wenkert D, et al. Herpes Zoster Reactivation in Patients With Rheumatoid Arthritis: Analysis of Disease Characteristics and Disease-Modifying Antirheumatic Drugs. Arthritis Care Res (Hoboken) 2015;67(12):1671-8.

[49] Chen CM, Chen HJ, Chen WS, Lin CC, Hsu CC, Hsu YH. Clinical effectiveness of influenza vaccination in patients with rheumatoid arthritis. Int J Rheum Dis 2018;21(6):1246-53.

[50] Park JK, Lee MA, Lee EY, Song YW, Choi Y, Winthrop KL, et al. Effect of methotrexate discontinuation on efficacy of seasonal influenza vaccination in patients with rheumatoid arthritis: a randomised clinical trial. Ann Rheum Dis 2017;76(9):1559-65. 
Figure 1 : Prise en charge de la fragilité. Adapté de Sugihara et al [29] 


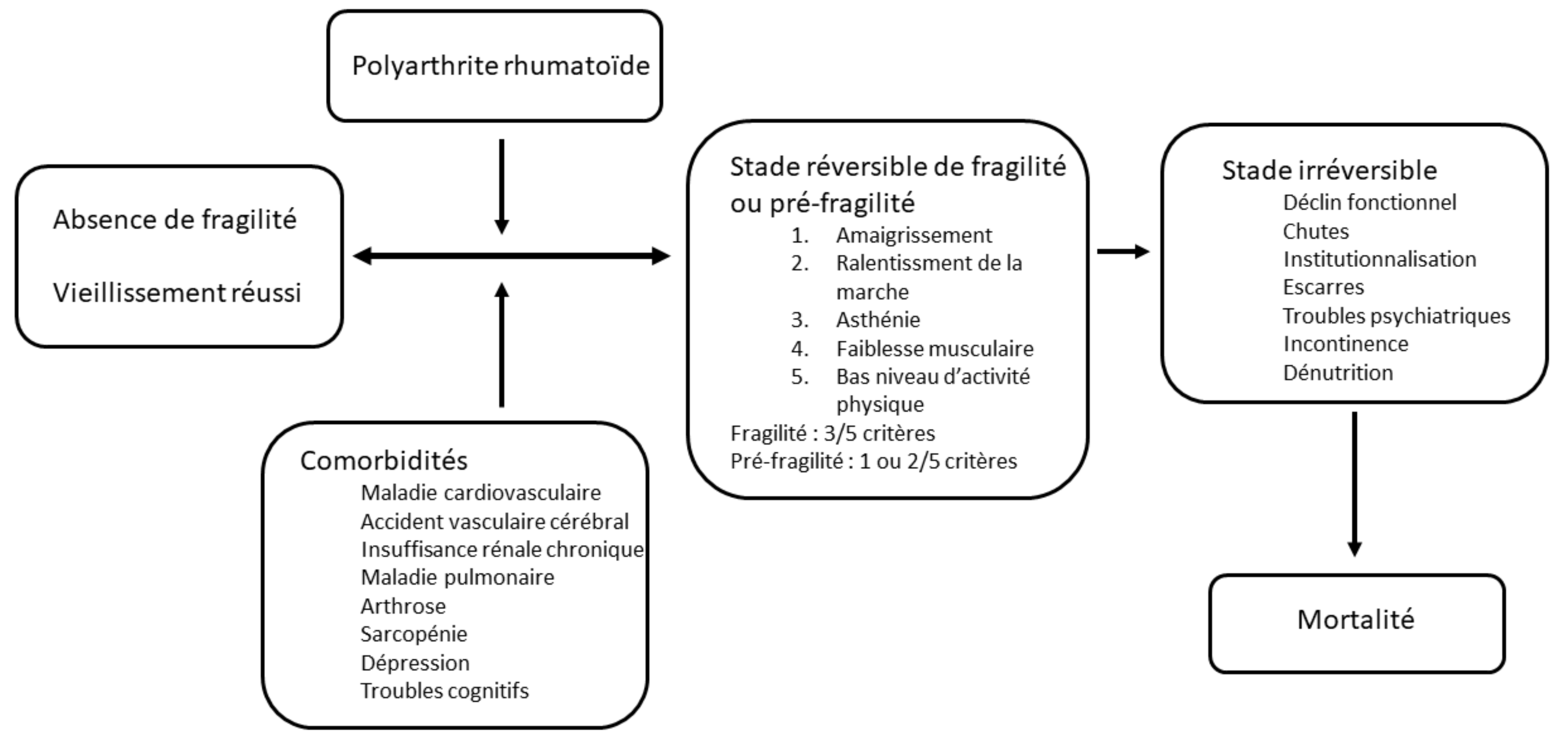

